\title{
Effect of Levosimendan Infusion 24 Hours Before CABG Surgery in Patients with Impaired Left Ventricular Function on the Need for Postoperative IABP
}

\author{
Sherif Nasr ${ }^{1}$, Hesham Alkady ${ }^{2,}$,, Ahmed Saber $^{2}$, Ahmed Elsharkawy ${ }^{2}$, Eman Mahmoud $^{3}$ \\ ${ }^{1}$ Department of Cardiothoracic Surgery, Fayoum University, Fayoum, Egypt \\ ${ }^{2}$ Department of Cardiothoracic Surgery, Cairo University, Cairo, Egypt \\ ${ }^{3}$ Department of Cardiology, Fayoum University, Fayoum, Egypt
}

Email address:

heshamalqady@cu.edu.eg (H. Alkady)

${ }^{*}$ Corresponding author

\section{To cite this article:}

Sherif Nasr, Hesham Alkady, Ahmed Saber, Ahmed Elsharkawy, Eman Mahmoud. Effect of Levosimendan Infusion 24 Hours Before CABG Surgery in Patients with Impaired Left Ventricular Function on the Need for Postoperative IABP. International Journal of Cardiovascular and Thoracic Surgery. Vol. 5, No. 2, 2019, pp. 51-55. doi: 10.11648/j.ijcts.20190502.15

Received: May 4, 2019; Accepted: June 2, 2019; Published: June 12, 2019

\begin{abstract}
Background: Due to their unfavorable outcome, patients with impaired left ventricular function undergoing CABG surgery (LVEF $\leq 35 \%$ ) are in a real need for optimization of their preoperative status in order to achieve the best possible results. In this retrospective comparative study we analysis our results in patients with impaired LV function after using Levosimendan as a continuous infusion 24 hours prior to CABG surgery regarding the need for postoperative IABP. Patients and methods: We included in this study 103 patients with LVEF $\leq 35 \%$ that underwent coronary artery bypass grafting with or without repair of ischemic mitral regurgitation and received Levosimendan infusion 24 hours before surgery in the period between January 2016 and January 2019 in 2 hospitals (Group A). These data were compared to another matched control group of 98 patients with similar conditions that were operated in the same hospitals over a previous period of 3 years but received no Levosimendan infusion preoperatively (Group B). Results: There was a statistically significant difference in the postoperative results in favor of group A regarding the need for IABP application (P-value $=0.013$ ). However there were no statistically significant differences between both groups in concern of duration of inotropic support ( $\mathrm{P}$-value $=0.40$ ), duration of mechanical ventilation ( $\mathrm{P}$-value $=0.30)$, total ICU $(\mathrm{P}$-value $=0.20)$ and hospital stays $(\mathrm{P}$-value $=0.40)$, incidence of postoperative atrial fibrillation $(\mathrm{P}$-value $=0.50)$, incidence of major adverse effects, and in-hospital mortality $(\mathrm{P}$-value $=$ 0.20). There was only one in-hospital mortality in each group. Conclusion: According to our study, infusion of Levosimendan 24 hours prior to CABG surgery in patients with impaired left ventricular contractility is safe and effective in reducing the need of IABP application. However Levosimendan infusion did not affect significantly postoperative coarse, incidence of major adverse effects, and in-hospital mortality.
\end{abstract}

Keywords: CABG Surgery, Levosimendan Infusion, Intra-aortic Balloon Counter-pulsation Pump

\section{Introduction}

With advances in health care services whether in diagnostic tools or therapeutic options, more risky patients especially those with impaired left ventricular (LV) function are referred to surgery. Unfortunately these patients represent a problematic population due to their unfavorable outcome especially for the fact that there is a $19 \%$ increase in the odds of death with each 10 -unit decrease in preoperative LV ejection fraction (LVEF) according to the Society of Thoracic Surgeons National Adult Cardiac Surgery Database. [1] Therefore such patients are in a real need for optimization of their preoperative status in order to achieve the best possible results. Hence the prophylactic use of inotropic agents especially Levosimendan has been suggested as a pharmacological preparation to improve postoperative outcomes in those patients. [2-3] In this retrospective 
comparative study we analysis our results in patients with impaired LV function after using Levosimendan as a continuous infusion 24 hours prior to CABG surgery regarding the need for postoperative IABP.

\section{Patients and Methods}

We included in this study 103 patients with LVEF $\leq 35 \%$ that underwent coronary artery bypass grafting with or without repair of ischemic mitral regurgitation and received Levosimendan infusion 24 hours before surgery in the period between January 2016 and January 2019 in 2 hospitals (Group A). These data were compared to another matched control group of 98 patients with similar conditions that were operated in the same hospitals over a previous period of 3 years but received no Levosimendan infusion preoperatively (Group B). An informed consent was obtained from all patients and an institutional review board was obtained.

Patients with recent myocardial infarction (MI) within 2 weeks, valve lesions other than the mitral valve, chronic renal failure (serum creatinine $>2 \mathrm{mg} / \mathrm{dl}$ ) and prior cardiac surgery were not included in the study. A myocardial viability study using Thalium perfusion scan or Dobutamine stress echocardiography was performed for all patients preoperatively to ensure the presence of viable myocardium for revascularization.

Group A patients were admitted one day before surgery to the ICU where invasive monitoring of arterial blood pressure and central venous pressure was established together with monitoring of urine output, pulse oximetry and electrocardiograpy (ECG). Levosimendan (Simdax, Orion Pharma, Espoo, Finland) was administered as a continuous infusion at a dose of $0.1 \mu \mathrm{g} / \mathrm{kg} / \mathrm{min}$ for 24 hours without a loading dose.

All patients received the same anaesthetic protocol and were operated through full median sternotomy. Patients without concomitant mitral valve repair were operated without the help of heart lung machine (Offpump) which is routine at our hospitals. All CABG procedures were performed by the same surgeons with the use of the left internal mammary artery (LIMA) as a conduit for the left anterior descending artery (LAD) and saphenous vein grafts (SVGs) for the other coronary vessels. Mitral valve repair was done in all cases with a remodeling annuloplasty ring (Edwards Lifesciences Physio 1 ring). In the ICU the haemodynamic parameters, duration of inotropic support, need for IABP, duration of mechanical ventilation, and length of stay were recorded in both groups. In addition other data of the postoperative course were collected including total hospital stay, occurrence of major adverse events and operative mortality (death within 30 days postoperatively or during the same hospitalization). Major adverse events were defined by postoperative myocardial infarction (CK-MB levels $>125 \mathrm{IU} / \mathrm{l}$ during the first 72 hours with development of a new Q wave or left bundle branch block in the ECG), new renal impairment (serum creatinine levels $>2 \mathrm{mg} / \mathrm{dl}$ or twice the preoperative creatinine) and postoperative stroke (new neurological deficit persisting more than 24 hours).

\section{Statistical Analysis}

Data were coded and then analyzed using the SPSS statistical software (IBM SPSS Statistics, version 19). Continuous variables were expressed as mean \pm standard deviation while categorical variables were expressed as numbers and percentages. The Mann-Whitney U-test was used to compare the continuous variables and the $\chi 2$ test or Fisher's exact test was used to compare the categorical data. Paired analysis and ANOVA for repeated measurements were used to detect differences between-groups. A P-value $<0.05$ was considered statistically significant.

\section{Results}

There were no statistically significant differences in both groups regarding preoperative patient characteristics including; age, gender, risk factors, EuroSCORE II, LVEF, percentage of scar tissue to viable myocardium and significant mitral regurgitation necessitating repair (Grade III-IV). Table 1.

Table 1. Preoperative patient characteristics (LVEF; left ventricular ejection fraction).

\begin{tabular}{llll}
\hline Preoperative patient characteristics & Group A $(\mathbf{n}=\mathbf{1 0 3})$ & Group B $(\mathbf{n}=\mathbf{9 8})$ & P-value \\
\hline Age & $57 \pm 11.3$ & $55 \pm 12.6$ & 0.51 \\
Gender & & & 0.65 \\
Males & $95(92.3 \%)$ & $93(94.9 \%)$ & 0.42 \\
Risk factors & & & 0.53 \\
Dyslipidemia & $55(53.4 \%)$ & $48(49 \%)$ & 0.60 \\
Systemic hypertension & $70(68 \%)$ & $62(63 \%)$ & 0.35 \\
Diabetes Mellitus & $67(65 \%)$ & $58(59 \%)$ & 0.62 \\
Smoking history & $83(80.6 \%)$ & $71(72 \%)$ & 0.70 \\
EuroSCORE II & $2.8 \pm 1.1$ & $2.5 \pm 1.3$ & 0.32 \\
LVEF (\%) & $30 \pm 4.6$ & $31 \pm 3.5$ & 0.27 \\
Scar tissue/ viable myocardium (\%) & $25 \pm 7$ & $28 \pm 5.8$ & 0.40 \\
Mitral regurgitation (III-IV) & $29(28.1 \%)$ & $22(22.5 \%)$ & $13(13.26 \%$ \\
Left main disease & $11(10.68 \%)$ & & \\
\hline
\end{tabular}

Levosimendan infusion could be completed in all patients of group A without the occurrence of hemodynamic 
instabilities or need for vasopressors.

Similarly operative data were statistically similar in both groups in concern of the total operative time, cardiopulmonary bypass (CPB) and cross clamp (CC) times in cases with concomitant mitral valve repair, average number of grafts, occurrence of arrhythmia whether atrial or ventricular, and conversion to on-pump bypass. Table 2 .

Table 2. Operative data (CPB; cardiopulmonary bypass, CC; cross-clamp).

\begin{tabular}{llll}
\hline Operative data & Group A (n= 103) & Group B (n=98) \\
\hline Total operative time (hours) & $3.1 \pm 1.2$ & $3.6 \pm 1.1$ & P-value \\
CPB time (in cases with mitral repair) (min) & $82 \pm 13$ & $78 \pm 8$ & 0.50 \\
CC time (in cases with mitral repair) (min) & $70 \pm 11$ & $68 \pm 12$ & 0.45 \\
Average number of grafts & $3.2 \pm 0.4$ & 0.70 \\
Occurrence of arrhythmia & & $32(32.6 \%)$ \\
Atrial & $26(25.2 \%)$ & $10(10.2 \%)$ & 0.20 \\
Ventricular & $8(7.76 \%)$ & $3(3.06 \%)$ \\
Conversion to on-pump bypass & $2(1.9 \%)$ & 0.35 \\
\hline
\end{tabular}

On the other hand there was a statistically significant difference in the postoperative results in favor of group A regarding the need for IABP application (P-value $=0.013$ ). However there were no statistically significant differences between both groups in concern of duration of inotropic support, duration of mechanical ventilation, total ICU and hospital stays, incidence of postoperative atrial fibrillation (AF), incidence of major adverse effects, and in-hospital mortality. Table 3.

Table 3. Postoperative results (MI; myocardial infarction, AF; atrial fibrillation; IABP, intra-aortic counter-pulsations pump).

\begin{tabular}{llll}
\hline Postoperative results & Group A (n= 103) & Group B (n=98) & P-value \\
\hline Need for IABP application & $1(0.97 \%)$ & $9(9.2 \%)$ & $4.3 \pm 1.2$ \\
Duration of inotropic support (days) & $3.5 \pm 1.5$ & $32 \pm 12$ & 0.013 \\
Duration of mechanical ventilation (hours) & $28 \pm 9$ & & 0.40 \\
Adverse events & $1(0.97 \%)$ & $1(1.02 \%)$ & $1(1.02 \%)$ \\
MI & 0 & $3(3.06 \%)$ & 0.30 \\
Stroke & $2(1.94 \%)$ & $25(25.5 \%)$ \\
Renal failure & $33(32 \%)$ & $5.3 \pm 1.3$ & 0.35 \\
Postoperative Arrhythmia (AF) & $4.8 \pm 1.7$ & $10 \pm 2.1$ \\
Total ICU stay (days) & $9.1 \pm 2.5$ & $1(1.02 \%)$ \\
Total hospital stay (days) & $1(0.97 \%)$ & 0.40 \\
In-hospital mortality & & 0.20 \\
\hline
\end{tabular}

The total health care service coasts of all patients in both groups were similar with no statistically significant difference. There was only one in-hospital mortality in each group. In group $\mathrm{A}$, one patient developed malignant intractable ventricular fibrillation on the second postoperative day and died suddenly despite normal serum electrolytes and without postoperative ECG changes. In group B, one patient developed severe low cardiac output syndrome immediately after CABG surgery and mitral valve repair which did not respond to maximum inotropic support and IABP application.

\section{Discussion}

Levosimendan (pyridazinone-dinitrite) is a member of a new group of inotropic agents known as the calcium sensitizers that are recently used in cardiac surgery to increase myocardial contractility especially in patients with impaired LV function. [4] Levosimendan has 3 unique vasoactive inotropic actions; it reduces the calcium-binding coefficient of troponin $\mathrm{C}$ enhancing myocardial contraction but with lower intracellular calcium concentration requirements in contrast to traditional inotropic agents (catecholamines and phosphodiesterase inhibitors) that increase cAMP resulting in increased calcium current into myocytes during systole. In addition Levosimendan opens adenosine triphosphate-sensitive potassium channels on vascular smooth muscle cells and in cardiac mitochondria. This results in arterial, coronary and venous vasodilatation decreasing preload and afterload in the systemic and pulmonary circulations as well as in mitochondrial activation acting in synergism with calcium sensitization to improve myocardial performance. [5] These actions are shown in some studies to be superior to those of traditional inotropic agents in improving surgical outcome especially in patients with impaired LV contractility since they are not associated with increased myocardial oxygen demand or decreased diastolic function. [6] However controversies do exist till now regarding the efficacy of prophylactic use of Levosimendan in cardiac surgery even in meta-analysis studies. [7-9] But when reviewing most of studies showing no benefits of the preoperative use of Levosimendan before cardiac surgeries we notice that most of the patients included in these studies received Levosimendan either shortly before 
surgery or in the operating room and with a loading dose. Also some of these studies included patients with normal ventricular function leaving no real action for Levosimendan in improving myocardial contractility and thus surgical outcome. [10]

Our study included a larger sample size of patients with impaired LV function needing CABG surgery with or without concomitant ischemic mitral repair and receiving preoperative Levosimendan than previous similar studies which were underpowered by small patient cohort. [11] In addition, in our series we have chosen the strategy of giving Levosimandan as a continuous infusion at a rate of $0.1 \mu \mathrm{g} / \mathrm{kg} / \mathrm{min}$ without loading 24 hours before surgery to avoid the adverse effects of Levosimendan; namely hypotension and arrhythmias noted when giving the drug as an intravenous bolus or infusion at higher rates. [12] At that infusion rate the drug itself reaches a linear concentration in blood after 4 hours while its active metabolite, OR-1896, continues in blood to a peak level about 48 hours and achieves a half-life of about 80 hours after the end of the infusion, covering the most critical postoperative period after CABG surgery until complete recovery of the ischemic myocardium. [13] This strategy appears successful in avoiding the adverse effects of the drug since none of group A patients experienced these effects.

In our study we have focused on surgical outcome especially in-hospital mortality, ICU and hospital stays as well as the need for IABP insertion which is the main goal and what really matters for cardiac surgeons in high risk patients with low EF undergoing CABG surgery. According to our results administration of Levosimendan as a continuous infusion 24 hours before surgery was significantly associated with the reduction in the need for IABP but was not significantly associated with reduction in other postoperative parameters that appear to be more related to the expected course of such high risk patients after CABG surgery.

Although the use of IABP prior to CABG surgery in highrisk patients especially those with low LVEF has been shown in many studies to improve surgical outcomes in terms of inhospital mortality, postoperative hemodynamic parameters and ICU stay. Yet IABP application is in general an invasive procedure that might be associated with some adverse effects like limb ischemia and arterial dissection. [14] On the other hand the coasts of Levosimendan infusion and insertion of an IABP set are similar and the increased coasts of preoperative admission to ICU for one day to receive the infusion are compensated postoperatively if the patient needed IABP which will remain for at least 48 hours before being removed.

Lastly our study included a large number of CABG cases that were operated upon without the heart lung machine (74 out of 103 patients). In all these cases we did not experience any complications related to Levosimendan infusion like hypotension and arrhythmia. Actually Levosimendan was shown in some studies to improve hemodynamics when given in small doses in patients undergoing Offpump CABG surgery. [15-16]

\section{Conclusion}

According to our study, infusion of Levosimendan 24 hours prior to CABG surgery in patients with impaired left ventricular contractility is safe and effective in reducing the need of IABP application. However Levosimendan infusion did not affect significantly postoperative coarse, incidence of major adverse effects, and in-hospital mortality.

\section{References}

[1] Shahian DM, O’Brien SM, Filardo G, Ferraris VA, Haan CK, Rich JB, Normand SL, DeLong ER, Shewan CM, Dokholyan RS, Peterson ED, Edwards FH, Anderson RP; Society of Thoracic Surgeons Quality Measurement Task Force. The Society of Thoracic Surgeons 2008 cardiac surgery risk models: Part 1-coronary artery bypass grafting surgery. Ann Thorac Surg 2009; 88: S2-22.

[2] Harrison RW, Hasselblad V, Mehta RH, Levin R, Harrington $\mathrm{RA}$, Alexander JH. Effect of levosimendan on survival and adverse events after cardiac surgery: a meta-analysis. J Cardiothorac Vasc Anesth 2013; 27: 1224-32.

[3] Kolseth SM, Nordhaug DO, Stenseth R, Sellevold O, Kirkeby-Garstad I, Wahba A. Prophylactic treatment with levosimendan: a retrospective matched-control study of patients with reduced left ventricular function. Eur J Cardiothorac Surg. 2009 Dec; 36: 1024-30.

[4] Leppikangas H, Järvelä K, Sisto T, Maaranen P, Virtanen M, Lehto P, Karlsson S, Kööbi T, Lindgren L. Preoperative levosimendan infusion in combined aortic valve and coronary bypass surgery. Br J Anaesth. 2011 Mar; 106: 298-304.

[5] Raja SG, Rayen BS. Levosimendan in Cardiac Surgery: Current Best Available Evidence. Ann Thorac Surg 2006; 81: $1536-46$.

[6] Landoni G, Biondi-Zoccai G, Greco M, Greco T, Bignami E, Morelli A, Guarracino F, Zangrillo A. Effects of levosimendan on mortality and hospitalization. A metaanalysis of randomized controlled studies. Crit Care Med 2012; 40: 634-46.

[7] Lim JY, Deo SV, Rababa'h A, Altarabsheh SE, Cho YH, Hang D, McGraw M, Avery EG, Markowitz AH, Park SJ. Levosimendan reduces mortality in adults with left ventricular dysfunction undergoing cardiac surgery: a systematic review and metaanalysis. J Card Surg 2015; 30: 547-54.

[8] Mehta RH, Leimberger JD, van Diepen S, Meza J, Wang A, Jankowich R, Harrison RW, Hay D, Fremes S, Duncan A, Soltesz EG, Luber J, Park S, Argenziano M, Murphy E, Marcel R, Kalavrouziotis D, Nagpal D, Bozinovski J, Toller W, Heringlake M, Goodman SG, Levy JH, Harrington RA, Anstrom KJ, Alexander JH; LEVO-CTS Investigators. Levosimendan in patients with left ventricular dysfunction undergoing cardiac surgery. N Engl J Med 2017; 376: 2032 42.

[9] Elbadawi A, Elgendy IY, Saad M, Megaly M, Mentias A, Abuzaid AS, Shahin HI, Goswamy V, Abowali H, London B. Meta-Analysis of Trials on Prophylactic Use of Levosimendan in Patients Undergoing Cardiac Surgery. Ann Thorac Surg 2018 Mar 21. 
[10] Molfetta PD, Gregorini R, Fiore C, Santarpino G. Is There Still Room For The Prophylactic Use Of Levosimendan In Cardiac Surgery? Ann Thorac Surg 2018.

[11] Anastasiadis K, Antonitsis P, Vranis K, Kleontas A, Asteriou C, Grosomanidis V, Tossios P, Argiriadou H. Effectiveness of prophylactic levosimendan in patients with impaired left ventricular function undergoing coronary artery bypass grafting: a randomized pilot study. Interactive CardioVascular and Thoracic Surgery 2016; 1-8.

[12] Toller W, Heringlake M, Guarracino F, Algotsson L, Alvarez J, Argyriadou H, Ben-Gal T, Černý V, Cholley B, Eremenko A, Guerrero-Orriach JL, Järvelä K, Karanovic N, Kivikko M, Lahtinen P, Lomivorotov V, Mehta RH, Mušič Š, Pollesello P, Rex S, Riha H, Rudiger A, Salmenperä M, Szudi L, Tritapepe L, Wyncoll D, Öwall A. Preoperative and perioperative use of levosimendan in cardiac surgery: European expert opinion. Int J Cardiol. 2015; 184: 323-36.

[13] Kivikko M, Antila S, Eha J, Lehtonen L, Pentikäinen PJ.
Pharmacokinetics of levosimendan and its metabolites during and after a 24-hour continuous infusion in patients with severe heart failure. Int J Clin Pharmacol Ther 2002; 40: 465-71.

[14] Pilarczyk K, Boening A, Jakob H, Langebartels G, Markewitz A, Haake N, Heringlake M, Trummer G. Preoperative intraaortic counterpulsation in high-risk patients undergoing cardiac surgery: a meta-analysis of randomized controlled trials. Eur J Cardiothorac Surg 2016; 49: 5-17.

[15] Kandasamy A, Simon HA, Murthy P, Annadurai M, Ali MM, Ramanathan G. Comparison of levosimendan versus dobutamine in patients with moderate to severe left ventricular dysfunction undergoing off-pump coronary artery bypass grafting: a randomized prospective study. Ann Card Anaesth 2017; 20: 200.

[16] Barisin S, Husedzinovic I, Sonicki Z, Bradic N, Barisin A, Tonkovic D. Levosimendan in off-pump coronary artery bypass: a four-times masked controlled study. J Cardiovasc Pharmacol 2004; 44: 703-8. 